\title{
Peripheral blood neutrophils are hyperresponsive to IL-8 and Gro-a in cryptogenic fibrosing alveolitis
}

\author{
P.C. Glynn, E.M. Henney, I.P. Hall
}

\begin{abstract}
Peripheral blood neutrophils are hyperresponsive to IL-8 and Gro- $\alpha$ in cryptogenic fibrosing alveolitis. P.C. Glynn, E.M. Henney, I.P. Hall. C ERS Journals Ltd 2001. ABSTRACT: Cryptogenic fibrosing alveolitis (CFA) is characterized by increased pulmonary recruitment of peripheral blood neutrophils (PBNs) by interleukin (IL)-8 and other chemotactic mediators. This study investigated whether, in CFA, the PBN motility response is primed by IL-8 and growth-related oncogene (Gro)- $\alpha$, as demonstrated in other neutrophilic inflammatory diseases, and whether the motility response of PBNs to IL-8 and Gro- $\alpha$ can be abrogated using a selective antagonist for the neutrophil receptor for IL-8 and Gro- $\alpha$, CXCR2.

The percentage of PBNs to undergo shape change (\%SC), spontaneously and in response to IL-8 and Gro- $\alpha$, was measured in patients with CFA $(n=10)$ and controls $(n=10)$, and the effect of the CXCR2 antagonist SB272844 studied. Plasma levels of IL-8, and Gro- $\alpha$ were measured using an enzyme-linked immunosorbent assay (ELISA).

The \%SC of unstimulated PBNs and the potency of Gro- $\alpha$ and IL-8 to produce neutrophil polarization was greater in CFA than in controls; dose which produces $50 \%$ of maximal effect (EC50) of IL-8 was 3.6 $\pm 0.7 \mathrm{nM}$ for CFA versus $6.3 \pm 1.0 \mathrm{nM}$ for controls; $p<0.05$. SB272844 inhibited Gro- $\alpha$ induced but not IL-8 induced neutrophil shape change (equilibrium constant (KD) $123 \pm 18 \mathrm{nM}$ ). Plasma concentrations of Gro- $\alpha$ were increased in patients with CFA. PBNs are spontaneously activated and undergo a greater motility response to IL-8 and Gro- $\alpha$ in CFA.

Interleukin-8 and growth-related oncogene- $\alpha$, circulating in substimulatory amounts in cryptogenic fibrosing alveolitis, may prime the peripheral blood neutrophils motility response, thus increasing their capacity for migration to the lung. Selective CXCR2 antagonists may be useful to block the Gro- $\alpha$-induced priming response whilst preserving neutrophil functions mediated by CXCR1, the alternative neutrophil receptor for interleukin-8.
\end{abstract}

Eur Respir J 2001; 18: 522-529.

Division of Therapeutics, University Hospital of Nottingham, UK.

Correspondence: I.P. Hall

Division of Therapeutics

South block C Floor

Queens Medical Centre

University Hospital

Nottingham

NG7 2UH

UK

Fax: 441159422232

Keywords: Cryptogenic fibrosing alveolitis

CXCR2 antagonist

growth-related oncogene- $\alpha$

neutrophil shape change

SB272844

Received: June 262000

Accepted after revision May 42001
Cryptogenic fibrosing alveolitis (CFA) is a chronic interstitial lung disease involving both inflammatory and fibrotic components. The inflammatory component of the disease is dominated by neutrophils and alveolar macrophages. Whereas normally there are very few inflammatory cells resident in the bronchoalveolar tree of healthy individuals, neutrophils represent $10-20 \%$ of the inflammatory cells present in bronchoalveolar lavage fluid (BALF) in CFA [1, 2]. A number of neutrophil chemotactic mediators have been demonstrated in blood and BALF of patients with CFA, including interleukin (IL)-8 [3], leukotriene receptor $(\mathrm{LT}) \mathrm{B}_{4}[4]$, and granulocyte-colony stimulating factor (G-CSF) [5]. They are implicated in the pathogenesis of lung damage [6] and remodelling, either though enhanced neutrophil recruitment from the pulmonary vascular bed and subsequent oxidantinduced cytotoxicity [7], or in the case of IL-8, via the additional mechanism of regulation of angiogenesis [8], a key component of the fibrotic response. In another model of neutrophilic inflammatory disease, Crohn's disease, peripheral blood neutrophils (PBNs) demonstrate increased polarization in the bloodstream [9] and increased chemotactic response to IL-8. In CFA, however, there is thus far only indirect evidence of peripheral blood inflammatory cell priming, provided by a study demonstrating increased superoxide anion production in blood in CFA [10]. This study therefore investigated whether neutrophil polarization, the initial cytoskeletal rearrangement characteristic of migrating cells and essential to neutrophil chemotaxis, is increased in response to the neutrophil chemokines IL-8 and growth-related oncogene (Gro- $\alpha$ ) in CFA.

IL-8 and other members of the CXC chemokine family, chemotactic for neutrophils, signal through a family of seven transmembrane domain G-protein coupled chemokine receptors. CXCR2 is the specific receptor for Gro- $\alpha$, whereas IL- 8 binds with equal affinity to CXCR1 and CXCR2. Blocking the chemotactic signal to neutrophils through CXCR1 and CXCR2 using selective receptor antagonists is a potential therapeutic strategy in neutrophil-driven diseases such as CFA. A CXCR2 antagonist (SB225002) has been developed [11] and may have a therapeutic role to play in blocking neutrophil 
activation and migration from the blood to the lungs whilst partially preserving neutrophil function. This study therefore investigated the effects of an analogue of this antagonist, SB272844, on IL-8 and Gro- $\alpha$-induced neutrophil shape change (SC), to determine if it would be useful in controlling neutrophil migration.

\section{Methods}

\section{Subject recruitment}

All patients in the study had a clinical diagnosis of CFA as defined by the British Thoracic Society (BTS) national study [12]. They had to fulfil all three of the BTS criteria: "bilateral interstitial chest radiographic shadowing with bilateral basal inspiratory crackles and lung function parameters compatible with CFA - that is a restrictive and/or gas transfer defect" [12]. The chest radiographical appearances necessary for inclusion in the study were peripheral bilateral reticular, reticulonodular shadowing, or honeycomb appearance. High-resolution computed tomography (HRCT) was not a strict criteria for the study. However, in all cases where it was performed (8 out of 10), it confirmed bilateral, peripheral, subpleural reticular shadowing with limited or no ground-glass shadowing. A histological diagnosis was not necessary for inclusion in the study as only a minority of patients in the UK diagnosed with CFA have an open lung biopsy [12]. In accordance with the BTS recommendations, those with a defined connective tissue disease, evidence of extrinsic allergic alveolitis, sarcoidosis, pneumoconiosis, or bronchiectasis were excluded. None of the patients studied were receiving immunosuppressive treatment at the time of study or within the preceding 6 months. Forced expiratory volume in one second (FEV1), forced vital capacity (FVC), carbon monoxide diffusing capacity of the lung ( $T \mathrm{~L}, \mathrm{CO})$, carbon monoxide transfer coefficient $(K \mathrm{CO})$, and lung volumes were measured in each patient at the time of blood sampling. Healthy age and sex-matched volunteers from amongst staff members were recruited as a control group. Control subjects had no history of lung disease. Three of the 10 individuals in the CFA group and two of 10 in the control group were current smokers. Informed written consent was obtained from patients and controls and the study had the approval of the University Hospital of the National Health Service Trust Ethics Committee.

\section{Laboratory methods}

Fifty millilitres of venous blood was drawn from each individual using a sterile nonpyrogenic 19-gauge butterfly needle and was used immediately for extraction of a purified sample of neutrophils by discontinuous Percoll-gradient centrifugation [13]. A plasma sample was retained for cytokine estimation.

\section{Discontinuous Percoll-gradient centrifugation}

A stock of Percoll (100\% fine grade, Sigma-Aldrich, Poole, UK) was prepared in $0.9 \%$ saline in a ratio of 9:1. Fifty millilitres of blood was mixed with $4 \mathrm{~mL}$ of $3 \%$ sodium citrate anticoagulant and immediately centrifuged at $420 \times g$ for $20 \mathrm{~min}$ at room temperature. The platelet-rich plasma layer was removed and $6 \mathrm{~mL}$ of $6 \%$ Dextran was added to the remaining contents of the tube. The volume was made up to $50 \mathrm{~mL}$ with $0.9 \%$ saline and the solution was allowed to stand for $30 \mathrm{~min}$ for erythrocyte sedimentation to take place. The leukocyte-rich plasma was then aspirated and centrifuged at $420 \times g$ for $6 \mathrm{~min}$, and the pellet resuspended in 2-3 mL platelet poor plasma (PPP), prepared earlier by centrifuging the plasma sample at $1,200 \times g$ for $15 \mathrm{~min}$. Solutions of $51 \%$ and $42 \%$ Percoll were freshly prepared and the leukocyte solution was then overlaid on the $42 \%$ Percoll gradient, which was overlaid on the $51 \%$ solution in a polystyrene tube. The sample was centrifuged immediately at $275 \times g$ for $11 \mathrm{~min}$. The mononuclear cell band of cells was aspirated from the interface between the plasma and the $42 \%$ Percoll layer and the neutrophil band of cells aspirated from the interface between the $42 \%$ and $51 \%$ Percoll layers. The neutrophil solution was washed in a 50:50 solution of PPP and Hanks (Gibco Life Technologies, Paisley, Scotland, UK) without calcium or magnesium. Cell viability was assessed by trypan blue exclusion. A cytospin slide was made of each neutrophil suspension. Slides were fixed with methanol, stained with May-Grunwald Giemsa and examined by light microscopy, at a magnification of $\times 400$, to count the purity of each preparation. Cell solutions obtained were of high purity, with $96 \%$ neutrophils (95\% confidence interval 93.2-98.6\%).

\section{Neutrophil stimulation with agonists}

A $500 \mu \mathrm{L}$ sample of $2 \times 10^{6}$ neutrophils $\cdot \mathrm{mL}^{-1}$ resuspended in Hanks without calcium or magnesium, was incubated at $37^{\circ} \mathrm{C}$ in a water bath for $20 \mathrm{~min}$. An equal volume of $2.5 \%$ glutaraldehyde was added to fix the cells and the percentage that had undergone spontaneous SC in each sample was calculated using the methods described later. Paired neutrophil suspensions were incubated with a range of concentrations of IL-8 (Peprotech, London, UK) or Gro- $\alpha$ (Peprotech) for $20 \mathrm{~min}$ at $37^{\circ} \mathrm{C}$, and then fixed in the same manner. Simultaneously paired neutrophil suspensions were preincubated with a range of concentrations of the CXCR2 antagonist, SB272844 [11], or its vehicle control, $0.1 \%$ dimethylsulphoxide (DMSO), for $5 \mathrm{~min}$, followed by the addition of $10 \mathrm{nM}$ IL-8 or $10 \mathrm{nM}$ Gro- $\alpha$ and incubation for a further $20 \mathrm{~min}$. After fixation for $5 \mathrm{~min}$, the cells were spun down at $300 \times g$ for $5 \mathrm{~min}$ in sterile $3 \mathrm{~mL}$ polystyrene tubes, washed in phosphate-buffered saline (PBS) and resuspended in PBS for shape change evaluation. Where insufficient donor neutrophils were obtained to complete all the experiments listed, the experiment measuring neutrophil SC to Gro- $\alpha$ (with or without 
SB272844) was omitted. Because the neutrophils were fixed, samples could be stored for later evaluation.

\section{Neutrophil shape change evaluation}

Two methods were used to evaluate and compare percentage spontaneous and IL-8-induced neutrophil $\mathrm{SC}$; microscopic scoring (\%SCMS), and flow cytometry (\%SCFC). Correlation between the two methods of assessment of neutrophil SC was determined using linear regression to compare spontaneous $\%$ SCMS versus \%SCFC of neutrophils from all 20 donors and \%SCMS versus \%SCFC after a range of concentrations of IL-8 in 10 patients and six controls (microscopic scoring was not available for four controls). The Pearson's correlation coefficient was calculated and there was excellent correlation between the two methods, $\left.\mathrm{r}^{2}=0.98, \mathrm{p}<0.0001\right)$. Because of this, stored fixed neutrophil suspensions were not analysed by flow cytometry in all cases. Flow cytometry was only used to evaluate spontaneous neutrophil SC and the neutrophil SC response to IL- 8 in the 20 subjects studied. Microscopic scoring was used to evaluate neutrophil SC to Gro- $\alpha$, and the response to the CXCR2 antagonist SB272844. The two methods are described briefly.

\section{Microscopic scoring}

A variation of the microscopic scoring method of Hatson and ShiElds [14] was used. Fixed cells were mounted in a Neubauer haemocytometer. Cells were categorized as being round or polarized at $\times 125$ magnification under transmission illumination, counting a sample of $>200$ cells each time. The ratio of polarized cells to the total cell count was expressed as a percentage SC by \%SCMS.

\section{Flow cytometry}

The flow cytometric method described by COLE et al. [15] was the second method used. A FACScan flow cytometer was used to analyse forward and wide-angle scatter, using linear amplification for both parameters. For each sample, list mode data from 5,000 events were stored with subsequent off-line analysis using windmdi version 2.8 (Scripps Institute, CA, USA). After gating on neutrophils an upper boundary for the forward scatter of neutrophils without SC was set at 3 SD above the mean linear channel number, determined using the unimodal distribution of unstimulated cells. The proportion of stimulated cells showing greater forward scatter than the unstimulated was expressed as a percentage of the total cells (\%SC by flow cytometry: \%SCFC).

\section{Plasma cytokine concentrations}

Plasma samples obtained during the neutrophil isolation procedure were stored at $-80^{\circ} \mathrm{C}$ until subsequent analysis of cytokine concentrations. PPP was obtained by centrifuging the plasma samples at $1,200 \times \mathrm{g}$ for $10 \mathrm{~min}$ for measurement of Gro- $\alpha$ concentrations since Gro- $\alpha$ is present in platelets and released upon platelet activation [16]. Samples were analysed in duplicate for concentrations of tumour necrosis factor (TNF)- $\alpha$, IL-8 and Gro- $\alpha$ using commercially available Quantikine sandwich enzymelinked immunosorbent assays (ELISAs) (R\&D Systems, Abingdon, UK). The sensitivities of the assays according to the manufactures are 4.4, 10, and $10 \mathrm{pg} \cdot \mathrm{mL}^{-1}$, respectively.

\section{Analysis}

A sigmoid dose-response curve plotting \%SCFC against log IL-8 concentration was constructed for each donor and the dose which produced $50 \%$ of maximal effect (EC50) was calculated for the $\%$ SCFC to IL-8 of each paired neutrophil suspension. Unpaired t-tests were used to compare the mean EC50 values for $\%$ SCFC to IL-8 of patients to controls, and likewise, spontaneous \%SCFC. Plasma concentrations of cytokines were compared using the Mann-Whitney U-test for nonparametric data. Correlation between $\%$ SCFC and plasma Gro- $\alpha$, and IL-8 levels was determined using linear regression, calculating Pearson's correlation coefficient. The equilibrium dissociation constant (KD) for SB272844 was calculated in individual experiments from the inhibition curve using the equation [17]:

$$
[\mathrm{Af}] / \mathrm{FEC}_{0}=1+(\mathrm{FIC50} / \mathrm{KD})
$$

where $[\mathrm{Af}]$ is the fixed agonist concentration, FEC50 is the agonist concentration producing $50 \%$ of the response produced by the fixed agonist concentration, and FIC50 is the antagonist concentration that reduces the fixed maximum response by $50 \%$.

\section{Results}

\section{Volunteer characteristics}

Ten patients with CFA and 10 healthy controls were studied. The mean ages of the two groups were 66.8 and 53.3, respectively. All patients had clinical evidence of CFA and impairment of lung function consistent with CFA (table 1). The mean $T \mathrm{~L}, \mathrm{CO}$ and $\mathrm{KCO}$ were $52 \pm 5 \%$ and $76 \pm 7 \%$ predicted, respectively. Lung volumes revealed a restrictive pattern with a mean total lung capacity (TLC), residual volume (RV), and functional residual capacity (FRC) of $76 \pm 5 \%, \quad 55 \pm 7 \%$, and $72 \pm 5 \%$ of predicted, respectively. All patients had chest radiographs documenting bilateral reticular opacities and eight patients had HRCT confirming bilateral, peripheral, subpleural reticular shadowing, consistent with CFA and in only one case ground-glass shadowing. 
Table 1. - Lung function in patients $(n=10)$ with cryptogenic fibrosing alveolitis

\begin{tabular}{lcc}
\hline Parameter & Measured & Predicted \% \\
\hline FEV1 L & $2.3 \pm 0.2$ & $87 \pm 3.5$ \\
FVC L & $3.1 \pm 0.3$ & $90 \pm 3.6$ \\
TL,CO & $4.4 \pm 0.5$ & $52 \pm 4.9$ \\
$\quad \mathrm{mmol} \cdot \mathrm{min}^{-1} \cdot \mathrm{KPa}^{-1}$ & $1.1 \pm 0.1$ & $76 \pm 7.2$ \\
$\quad \mathrm{CCO}$ & & \\
$\quad \mathrm{mmol} \cdot \mathrm{min}^{-1} \cdot \mathrm{KPa}^{-1} \cdot \mathrm{L}^{-1}$ & $4.7 \pm 0.4$ & $76 \pm 5.0$ \\
TLC L & $1.3 \pm 0.1$ & $55 \pm 6.7$ \\
$\mathrm{RV} \mathrm{L}$ & $2.4 \pm 0.2$ & $72 \pm 5.0$ \\
FRC L &
\end{tabular}

Data are prsented as mean \pm SEM. FEV1: forced expiratory volume in one second; FVC: forced vital capacity; TL,CO: carbon monoxide diffusion capacity of the lung; KCO: carbon monoxide transfer coefficient; TLC: total lung capacity; RV: residual capacity; FRC: functional residual capacity.

\section{Baseline rates of neutrophil shape change}

Baseline rates of spontaneous neutrophil SC were significantly higher in the patient versus the control group: \% $\%$ SFC was $7.12 \pm 1.3 \%$ versus $3.7 \pm 0.8 \%$ $(\mathrm{p}<0.05)$ and $\%$ SCMS was $5.7 \pm 0.9 \%$ versus $2.5 \pm 0.4 \%$ $(\mathrm{p}<0.01)$.

\section{Effect of interleukin-8 on neutrophil shape change}

There was a dose-dependent increase in the \% $\%$ SCFS to IL-8 of PBNs from both healthy controls and patients with CFA (fig. 1). The CFA group had a significantly lower mean EC50 for $\% \mathrm{SCFC}$ to IL-8 than the control group $(3.6 \pm 0.7 \mathrm{nM}$ versus $6.3 \pm$ $1.0 \mathrm{nM}, \mathrm{p}<0.05$; fig. 1). The EC50 values were also lower in CFA than controls $(3.9 \pm 0.6 \mathrm{nM}$ versus $11.8 \pm 0.8 \mathrm{nM}, \mathrm{p}<0.05)$ when evaluated by microscopic scoring. The maximum response to IL-8 was similar in both groups $(87 \pm 2.5$ versus $81 \pm 2.7 \%, \mathrm{p}>0.05)$.

There was good correlation between the two

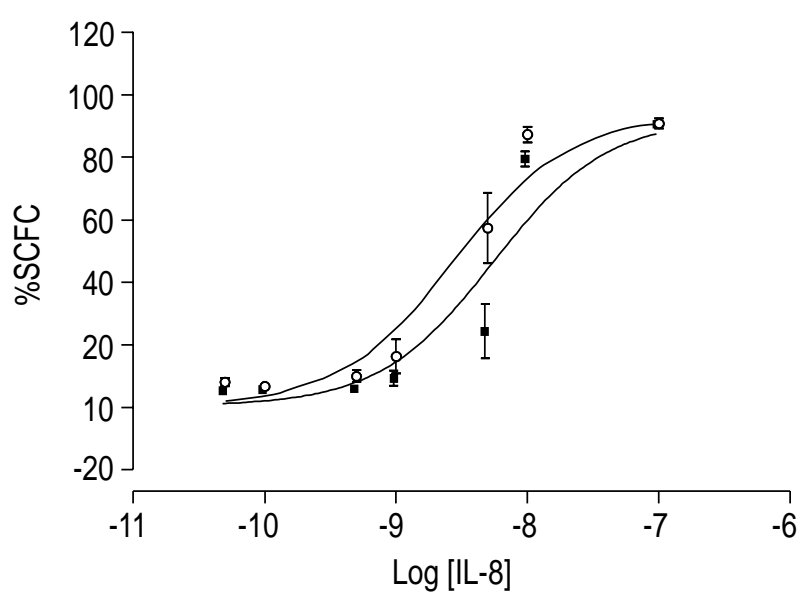

Fig. 1.-Percentage shape change measured by flow cytometry (\%SCFC) after stimulation with a range of concentrations of interleukin (IL)-8 in cryptogenic fibrosing alveolitis (CFA) patients $(\bigcirc)$ and controls $(\boldsymbol{\square})$. Data are presented as mean \pm SEM.

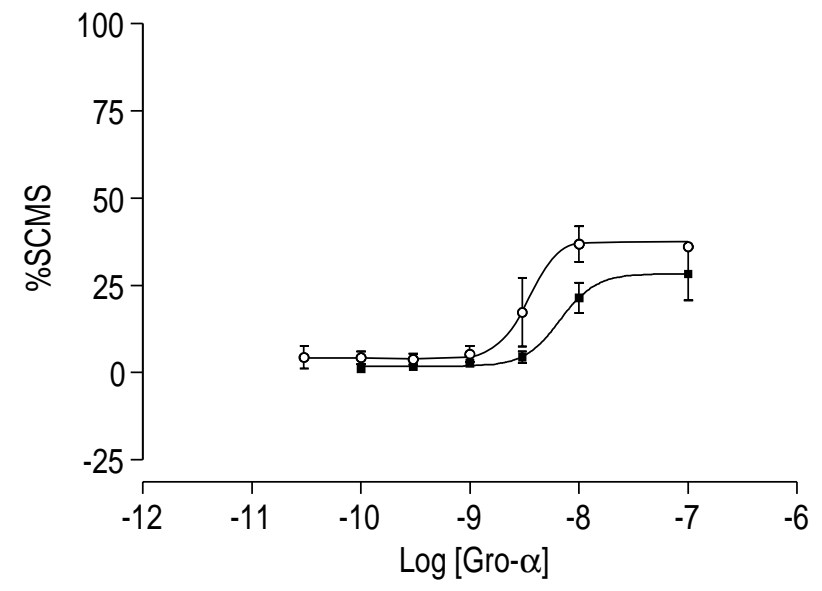

Fig. 2.-Percentage shape change measured by the microscopic scoring method ( $\% \mathrm{SCMS})$ after stimulation with a range of concentrations of growth-related oncogene (Gro- $\alpha$ ) in cryptogenic fibrosing alveolitis (CFA) patients $(\bigcirc)$ and controls $(\boldsymbol{\square})$. Data are presented as mean \pm SEM.

methods of evaluation for neutrophil SC (\%SCMS versus $\% \mathrm{SCFC}$ ) $\mathrm{r}^{2}=0.98, \mathrm{p}<0.0001$ (fig. 2). The latter method was only used to evaluate neutrophil SC response to IL-8.

Effect of growth-related oncogene- $\alpha$ on neutrophil shape change

There was a dose-dependent increase in the $\%$ SCMS of neutrophils to Gro- $\alpha$ (EC50 4.6 $\pm 0.6 \mathrm{nM}$, fig. 2). The polarizing effect of $10 \mathrm{nM}$ Gro- $\alpha$ on neutrophils extracted from patients with CFA $(n=5)$ and healthy controls $(n=7)$ was significantly different $(36.9 \%$ versus $21.4 \%, \mathrm{p}<0.05)$. However, the EC50 values of the dose-response curves did not differ significantly.

Effect of SB272844 on interleukin-8 and growthrelated oncogene- $\alpha$ mediated neutrophil shape change

Gro- $\alpha$ (10 nM)-induced neutrophil SC could be blocked by the CXCR2 antagonist SB272844 in a dose-dependent manner: inhibitory concentration of $50 \%$ (IC50) values were $31.6 \pm 3.9 \mathrm{nM}$ and $237 \pm 131$ and equilibrium constant $(\mathrm{KD})$ values were $1.2 \pm 0.2 \times 10^{-7}$ and $2.6 \pm 1 \times 10^{-7}$, in patient and control groups, respectively (fig. 3), i.e. in the range expected for a relatively potent selective CXCR2 antagonist. SB272844 failed to inhibit the neutrophil SC response induced by IL-8 in a concentration range expected for a selective competitive antagonist (IC50 values $>1 \mu \mathrm{M}$ ).

\section{Cytokine concentrations}

Plasma concentrations of TNF- $\alpha$ were below the level of detection of the assay $\left(4.4 \mathrm{pg} \cdot \mathrm{mL}^{-1}\right)$ in eight of 10 controls and eight of 10 patients. Plasma concentrations of IL-8 ranged from $12-30 \mathrm{pg} \cdot \mathrm{mL}^{-1}$ in the patient group and from undetectable to $18 \mathrm{pg} \cdot \mathrm{mL}^{-1}$ in 

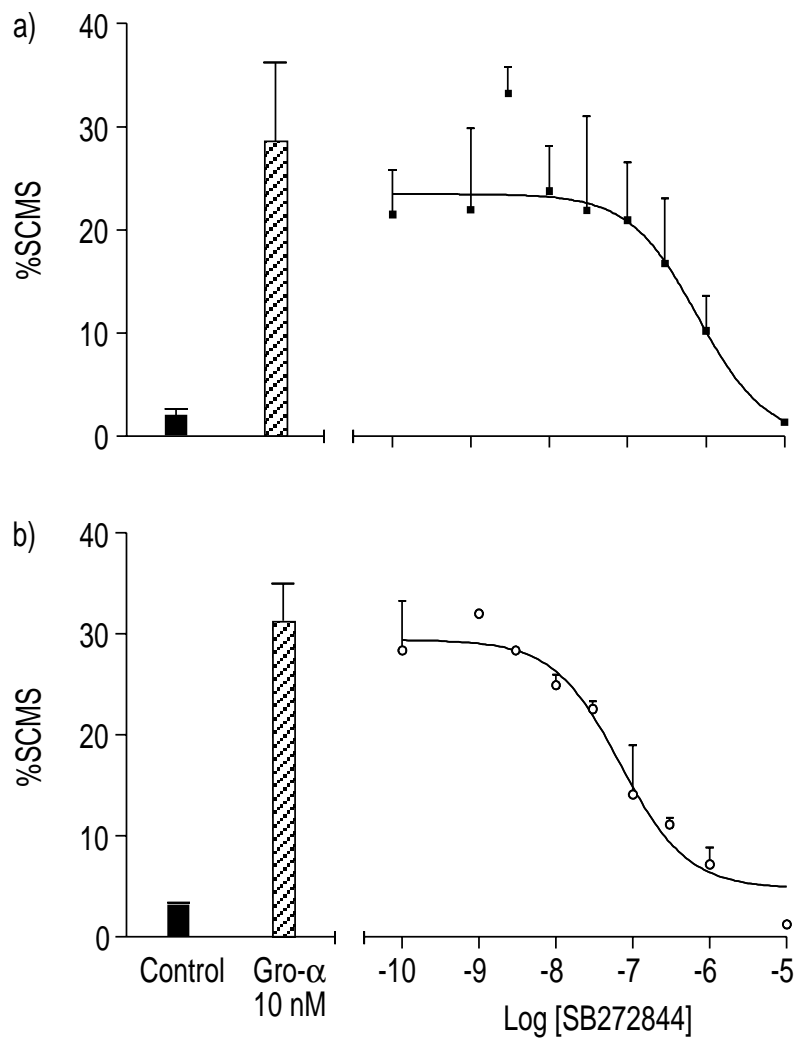

Fig. 3.-Percentage shape change measured by the microscopic scoring method (\%SCMS) after incubation with $10 \mathrm{nM}$ growthrelated oncogene (Gro- $\alpha$ ) in the presence or absence of a range of concentrations of the CXCR2 antagonist SB272844, in peripheral blood neutrophils. a) Controls $(n=5)$ and b) cryptogenic fibrosing alveolitis (CFA) patients $(n=5)$. Data are presented as mean \pm SEM.

the controls (median: $16.1 \mathrm{pa} \cdot \mathrm{mL}^{-1}$ versus $12.0 \mathrm{pa} \cdot \mathrm{mL}^{-1}$, respectively; $\mathrm{p}=0.07$ ). Gro- $\alpha$ was detected in all PPP samples from patients with a range of 63.5$1089 \mathrm{pg} \cdot \mathrm{mL}^{-1}$ and in 7 of 10 control samples with a range of $34.3-218 \mathrm{pg} \cdot \mathrm{mL}^{-1}$ (median $93.5 \mathrm{pa} \cdot \mathrm{mL}^{-1}$ versus $54.6 \mathrm{pa} \cdot \mathrm{mL}^{-1}$, respectively; $\mathrm{p}<0.05$, MannWhitney U-test; fig. 4).

\section{Discussion}

This study provided evidence that neutrophil polarization, the initial cytoskeletal rearrangement characteristic of migrating cells and essential to neutrophil chemotaxis, is increased both spontaneously and in response to the neutrophil chemokines IL- 8 and Gro- $\alpha$, in CFA. It is speculated that "priming" of the PBN response to IL-8 is a potential mechanism by which enhanced neutrophil recruitment to the lung may occur in this disease upon exposure to IL-8 or Gro- $\alpha$ in the pulmonary vascular bed or bloodstream. Such neutrophil priming may be a prerequisite to enhanced neutrophil chemotaxis to the inflamed lung in CFA, and may partly explain the reported positive correlation between IL-8 levels and percentage of BAL neutrophils observed in this condition [6]. Finally, elevated circulating levels of the neutrophil chemotactic protein Gro- $\alpha$ have been
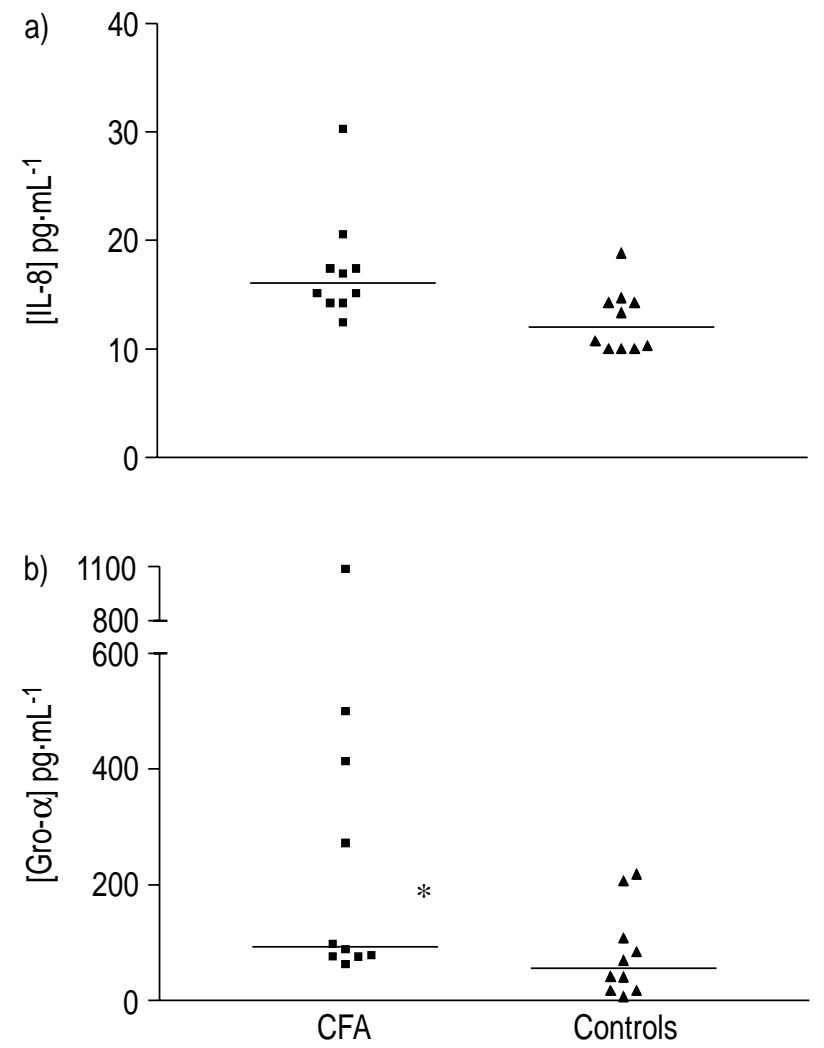

Fig. 4.-Plasma interleukin (IL)-8 and growth-related oncogene (Gro- $\alpha$ ) concentrations in controls and cryptogenic fibrosing alveolitis (CFA) patients. Median IL-8 concentrations are $16.1 \mathrm{pg} \cdot \mathrm{mL}^{-1}$ (range $12-30 \mathrm{pg} \cdot \mathrm{mL}^{-1}$ ) versus $12.0 \mathrm{pg} \cdot \mathrm{mL}^{-1}$ (range undetectable$\left.18 \mathrm{pg} \cdot \mathrm{mL}^{-1}\right)$, and median Gro- $\alpha$ concentrations are $93.5 \mathrm{pg} \cdot \mathrm{mL}^{-1}$ (range $63.5-1089 \mathrm{pg} \cdot \mathrm{mL}^{-1}$ ) versus $54.6 \mathrm{pg} \cdot \mathrm{mL}^{-1}$ (range undetectable$218 \mathrm{pg} \cdot \mathrm{mL}^{-1}$ ), in CFA and controls respectively. Median lines are shown. *: $\mathrm{p}<0.05$.

demonstrated for the first time in plasma in CFA and it is postulated that this may play a role in priming the neutrophil chemotactic response to the lung.

The inflammatory component of CFA in the lungs is dominated by neutrophils and alveolar macrophages, and neutrophils constitute $10-20 \%$ of the inflammatory cells present in BALF in CFA $[1,2]$. In animal models of neutrophilic alveolitis, PBN sequestration in the marginated pool in the pulmonary capillary bed is increased, and neutrophils move from this pool into the lung alveoli [18]. In an animal model of CFA, bleomycin-induced lung injury, radiolabelled PBNs could be detected in the lungs for up to three weeks [19]. To the best of the authors' knowledge, there has only been one study of neutrophil transit through the lungs in humans in CFA, in which ${ }^{111}$ Indium-labelled neutrophils were injected into the bloodstream and monitored during their movement into the lungs [20]. This study reported an increased retention time of labelled cells in the lungs in CFA compared to controls, and increased recovery of labelled neutrophils from the distal airways by BAL at $24 \mathrm{~h}$. The latter finding correlated with disease activity and mortality. These studies provide evidence that the increased neutrophil number in the lung in CFA is a result of increased retention of circulating 
peripheral blood neutrophils. The present authors have chosen to study PBN SC in CFA for a number of reasons. Firstly, there is prior indirect evidence of peripheral blood inflammatory cell "priming" in CFA, as demonstrated by increased superoxide anion production in blood [10]. As neutrophils are the most abundant cellular source of oxidants it was postulated that the observed oxidant imbalance in CFA may be coming from activated PBNs. Secondly, in another model of neutrophilic inflammatory disease, inflammatory bowel disease (IBD), PBNs demonstrate increased polarization and increased chemotactic response to IL- $8[9,21]$. However, there is currently no information available on the chemotactic response of PBNs in CFA. Neutrophil SC is a measure of neutrophil movement activation and has been shown to correlate well with chemotaxis measured using Boyden chambers [14], but has advantages over such methods, as it does not measure adhesive interactions and movements along surface bound chemotactic gradients (haptotaxis). Thirdly, PBNs are readily accessible without the use of invasive procedures. It can only be speculated that any neutrophil activation seen in PBNs would be even more marked in the marginated pool of neutrophils in the lung, were those neutrophils easily accessible. It has been shown that PBNs in CFA are hyperresponsive to IL- 8 and Gro- $\alpha$, and it is now speculated that they are being "primed" in the circulation by exposure to cytokines such as Gro- $\alpha$. Neutrophils are subject to priming when exposed to substimulatory concentrations of a number of pro-inflammatory cytokines in vivo or in vitro. Many of these are produced in the inflamed lung in CFA e.g. TNF- $\alpha$, IL-1 $\alpha$, IL-8, and platelet activating factor (PAF) and could be responsible for "priming" PBNs in vivo. TNF- $\alpha$ was not detected in either the present patient or control plasma samples, but this does not rule out the possibility of exposure to TNF- $\alpha$ bound to endothelial cells or monocytes on passing through the pulmonary circulation, which could not be measured. It was, however, demonstrated for the first time that plasma Gro- $\alpha$ concentrations are elevated in CFA, in concentrations that do not directly activate neutrophil chemotaxis, but are sufficiently high for neutrophil "priming". In vitro Gro- $\alpha$ primes the neutrophil chemotactic response to IL-8 [21] and the optimal concentration to do so is around $50 \mathrm{pg} \cdot \mathrm{mL}^{-1}$. All patients studied had plasma Gro- $\alpha$ concentrations $>50 \mathrm{pg} \cdot \mathrm{mL}^{-1}$ whereas only five out of 10 controls did. Gro- $\alpha$ levels did not correlate directly with the degree of observed spontaneous or IL- 8 - or Gro- $\alpha$-induced neutrophil SC, but the number of subjects was small and it is possible that correlations may have been seen in a larger study. This does not rule out "priming" of the neutrophil SC response by Gro- $\alpha$ and the apparent discrepancy may also be due to the contribution of other priming agents that were not measured, such as G-CSF, $\mathrm{LTB}_{4}$ or PAF [22]. Ashitani et al. [5] demonstrated G-CSF in BAL, but not blood in CFA, and $\mathrm{LTB}_{4}$ and PAF have not been measured in serum of patients with CFA to the best of the authors' knowledge. Based on the trend shown in this data, further study of a larger number of patients would be warranted to establish whether Gro- $\alpha$ plays a central role in priming neutrophil migration in CFA. An alternative explanation for the observed neutrophil hyperresponsiveness to IL- 8 and Gro- $\alpha$ in CFA could be upregulation of CXCR2 on circulating neutrophils. This possibility has not been excluded, but it is thought to be unlikely, as G-CSF is the only cytokine known to increase surface expression of CXCR2 and a previous study failed to detect G-CSF circulating in peripheral blood in CFA [5]. Circulating IL- 8 or Gro- $\alpha$ would be unlikely to produce receptor upregulation, since they are both known to downregulate CXCR 1 and 2 expression on neutrophils in vitro [23]. In vivo CXCR2 downregulation on PBNs in patients with sepsis occurred in the presence of 10-fold higher concentrations of IL-8 and two-fold higher concentrations of Gro- $\alpha$ than have been seen in CFA, and was associated with a decreased chemotactic response to Gro- $\alpha$ [24], which is the opposite to that seen in the present study. In the authors' opinions, therefore, it is also unlikely that CXCR2 has been downregulated on PBNs in the patients studied. Regarding the relevance of CFA PBN hyperresponsiveness to IL-8, there is certainly evidence that IL-8 is the major chemotactic protein implicated in neutrophil accumulation in CFA, and thereafter activation and release of mediators that may contribute to the pathogenesis of lung damage [7]. Messenger ribonucleic acid (mRNA) expression for IL-8 is induced in alveolar macrophages taken from individuals with CFA and correlates with neutrophil number in the BAL [3]. The responsiveness of lung alveolar macrophages (AMs) to stimuli appears to be increased by exposure to the inflammatory milieu of the CFA lung, and AMs taken from individuals with CFA, but not other fibrotic lung diseases such as sarcoidosis, are primed for IL-8 production in vitro [25]. IL-8 concentrations reported in BALF in two studies of CFA were in a range capable of producing only low levels of chemotaxis of healthy neutrophils in vitro, $0.24 \pm 0.1 \mathrm{nM}$ [25] and $0.09 \pm 0.01 \mathrm{nM}$ [6]. However, IL-8 levels did correlate with neutrophil numbers in BAL in these studies. In the assay of the present study, CFA PBNs did not undergo significantly higher rates of SC in response to subnanomolar concentrations of IL-8. However, in response to the 10 -fold higher BALF concentrations of IL-8 $(\sim 2 \mathrm{nM})$ demonstrated by CAR et al. [26], CFA PBNs could produce $\sim 40 \%$ higher rates of $\mathrm{SC}$ than control $\mathrm{PBNs}$ in vitro. In CFA, the factors controlling neutrophil load in the lung are obviously complex, as other chemotactic proteins in addition to IL-8 play a role in attracting neutrophils, and the neutrophil load is determined not only by neutrophil migration but also by neutrophil clearance. Gro- $\alpha$ is clearly less potent than IL- 8 in producing neutrophil $\mathrm{SC}$, and since it has not been measured previously in CFA, either in the lung or in blood, its role in this disease is not clear. However, concentrations of $1.7 \pm 0.4 \mathrm{ng} \cdot \mathrm{mL}^{-1} \quad(0.2 \pm 0.05 \mathrm{nM})$ have been demonstrated in BALF in ARDS, threetimes higher than the BALF IL-8 concentration in the same patients [27], and blocking Gro- $\alpha$ could significantly reduce neutrophil chemotactic activity of BALF in ARDS, suggesting a role for Gro- $\alpha$ in 
neutrophil migration in disease. Gro- $\alpha$ may also contribute to regulation of neutrophil number in the lung by delaying neutrophil apoptosis [28], thereby prolonging neutrophil lifespan in thelung. Serum IL-8 concentrations in the present study were relatively low compared to a previous one [6], suggesting that the present patients studied, which excluded those being treated for acute deterioration or worsening disease, may have had a relatively mild inflammatory component to their disease. However, there are no direct measures of the latter available, such as BALF neutrophil counts, for these patients. HRCT data is available for eight out of 10 patients, of whom only one demonstrated ground-glass shadowing. Lung function demonstrated a moderate impairment of TL,CO and RV (table 1), consistent with these patients having mild disease. Whether IL-8-induced chemotaxis is mediated by CXCR 1 or CXCR2 has been the subject of some debate, and is important if newly developed CXCR2 antagonists are to be developed for therapeutic purposes, to block neutrophil migration in disease states. Antibody blocking studies suggest that the majority of the chemotactic response is mediated by CXCR1 [29]. However, WhiTe et al. [11] suggested that IL-8-mediated neutrophil chemotaxis in vitro is mediated predominantly by CXCR 2 .

The present data indicates that IL- 8 can overcome the blockade of CXCR2 by SB272844 and continue to induce neutrophil SC, presumably by activating CXCR1. It is known that SB27284 is selective for CXCR2 because it inhibits Gro- $\alpha$ (a selective CXCR2 agonist)-induced neutrophil $\mathrm{SC}$ with a $\mathrm{K}_{\mathrm{D}}$ of $123 \mathrm{nM}$ in CFA. In contrast, IL-8 mediated SC was not inhibited by SB272844 at concentrations likely to be selective for CXCR2, and this response is, therefore, probably mediated by both CXCR 1 and CXCR2. Such redundancy in the chemokine receptor family is well described. The therapeutic implication of receptor redundancy is that both IL-8 receptors would have to be blocked to abolish the most potent chemotactic stimulus to neutrophils, IL-8, and at present there is no good CXCR1 antagonist available to do so. Although Gro- $\alpha$ is a less potent neutrophil chemoattractant than IL-8 in vitro, it is circulating in plasma in relatively higher quantities in CFA and may prime the neutrophil chemotactic response to IL-8. Therefore, a selective CXCR2 antagonist may be therapeutically useful to block the priming of PBNs and hence reduce the degree of neutrophil activation and migration to the lungs. However, based on the present study alone, there is not sufficient evidence that Gro- $\alpha$ is the sole agent involved in "priming" PBNs in CFA. While an advantage of selective CXCR2 blockade would be to partly preserve neutrophil function, since other neutrophil responses such as the respiratory burst [30] and exocytosis [31] can be maximally elicited by stimulation of CXCR1, and hence minimize the nonspecific immunosuppressive effects, it would appear that blocking both IL-8 receptors would be a more effective approach.

In summary, this study has demonstrated that peripheral blood neutrophils isolated from individuals with cryptogenic fibrosing alveolitis exhibit a spontaneous increase in shape change, and are primed to undergo a greater in vitro polarization response to the chemokines IL- 8 and Gro- $\alpha$. Substimulatory concentrations of interleukin- 8 and growth-related oncogene- $\alpha$ are present in the plasma of patients with crypotogenic fibrosing alveolitis, growth-related oncogene- $\alpha$ in relatively higher amounts in crypotogenic fibrosing alveolitis than in healthy controls. While one major drive to neutrophil recruitment to the lungs is the highly chemotactic activity of the lung milieu itself, a synergistic effect on neutrophil chemotaxis may result when such circulating "primed" neutrophils are exposed to their key motility activators in higher concentrations in the lung. While a dual approach to interleukin-8 receptor blockade would be necessary to abolish interleukin-8-induced neutrophil chemotaxis, an alternative approach that would preserve some aspects of normal neutrophil function and yet potentially counteract the growthrelated oncogene- $\alpha$-induced primed state of circulating neutrophils in crypotogenic fibrosing alveolitis could employ selective CXCR2 blockade.

Acknowledgements. The authors are grateful to T. Torphy, SmithKline Beecham (King of Prussia, USA), for the kind gift of the CXCR2 antagonist SB272844.

\section{References}

1. Watters LC, Scharz MI, Cherniack RM, et al. Idiopathic pulmonary fibrosis pretreatment bronchoalveolar lavage cellular constituents and their relationships with lung histopathology and clinical response to therapy. Am Rev Respir Dis 1987; 135: 696-704.

2. O'Donnell K, Keogh B, Cantin A, Crystal RG. Pharmacologic suppression of neutrophil component of alveolitis in idiopathic pulmonary fibrosis. Am Rev Respir Dis 1987; 136: 288-292.

3. Carre PC, Mortenson RL, King TE Jr, Noble PW, Sable CL, Riches DWH. Increased expression of the interleukin- 8 gene by alveolar macrophages in idiopathic pulmonary fibrosis. J Clin Invest 1991; 88: 1802-1810.

4. Wardlaw AJ, Hay H, Cromwell O, Collins JV, Kay AB. Leukotriens, LTC4 and LTB4, in bronchoalveolar lavage in bronchial asthma and other respiratory diseases. J Allergy Clin Immunol 1989; 84: 19-26.

5. Ashitani J-I, Mukae H, Taniguchi H, et al. Granulocyte-colony stimulating factor levels in bronchoalveolar lavage fluid from patients with idiopathic pulmonary fibrosis. Thorax 1999; 54: 1015-1020.

6. Ziegenhagen MW, Zabel P, Zissel G, Schlaak M, Muller-Quernheim J. Serum level of interleukin 8 is elevated in idiopathic pulmonary fibrosis and indicate disease activity. Am J Respir Crit Care Med 1998; 157: 762-768.

7. Cantin AM, North SL, Fells GA, Hubbard RC, Crystal RG. Oxidant-mediated epithelial cell injury in idiopathic pulmonary fibrosis. J Clin Invest 1987; 79: 1665-1673.

8. Keane MP, Arenberg DA, Lynch IIIJP, et al. The CXC chemokines, IL-8 and IP10, regulate angiogenic 
activity in idiopathic pulmonary fibrosis. $J$ Immunol 1997; 159: 1437-1443.

9. McCarthy DA, Rampton DS, Liu YC. Peripheral blood neutrophils in inflammatory bowel disease: morphological evidence of in vivo activation inactive disease. Clin Exp Immunol 1991; 86: 489-493.

10. Teramoto SF, Fukuchi Y, Uejima Y, Shu C-Y, Orimo H. Superoxide anion formation and glutathione metabolism of blood in patients with idiopathic pulmonary fibrosis. Biochem and Mol Med 1995; 55: 66-70.

11. White JR, Lee JM, Young PR, et al. Identification of a potent selective nonpeptide CXCR2 antagonist that inhibits interleukin-8 induced neutrophil migration. J Biol Chem 1998; 273: 10095-10098.

12. Johnston IDA, Prescott RJ, Chalmers JC, Rudd RM for the Fibrosing Alveolitis Subcommittee of the research committee of the British Thoracic Society. British Thoracic Society study of cryptogenic fibrosing alveolitis: current presentation and initial management. Thorax 1997; 52: 38-44.

13. Haslett C, Guthrie LA, Kopaniak MM, Johnston RB, Ghenson PM. Modulation of multiple neutrophil functions by preparative methods or trace concentrations of lipopolysaccharide. Am J Pathol 1985; 19: 101-110.

14. Hatson WS, Shields JM. Neutrophil leucocyte chemotaxis: a simplified assay for measuring polarising responses to chemotactic factors. J Immunol Methods 1985; 81: 229-237.

15. Cole AT, Garlick NM, Glavin AM, Hawkey CJ, Robins RA. A flow cytometric method to measure shape change of human neutrophils. Clin Sci 1995; 89: 549-554.

16. Takeuchi K, Higuchi T, Koike K. Chemokine production by human megakaryocytes derived from CD34-positive cord blood cells. Cytokine 1999; 11: 424434.

17. Lazareno S, Birdsall NJM. Estimation of antagonist Kd from inhibition curves in functional experiments: alternatives to Cheng-prusoff equation. Trends Pharmacol Sci 1989; 10: 442-447.

18. Brown GM, Brown DM, Donaldson K, Drost E, MacNee W. Neutrophil sequestration in rat lungs. Thorax 1995; 50: 661-667.

19. Haslett C, Shen AS, Feldsien DC, Allen D, Henson PM, Cherniack RM. ${ }^{111}$ Indium labeled Neutrophil migration into the lungs of bleomycin-treated rabbits assessed non-invasively by external scintigraphy. $\mathrm{Am}$ Rev Resp Dis 1989; 140: 756-763.

20. Worthen GS, Young SK, Ikle D, et al. Neutrophil localisation to the lung in idiopathic pulmonary fibrosis: a scintigraphic study. Am Rev Respir Dis 1990; 141: A651.
21. Brandt E, Muller-Alouf H, Desreumaux P, Woerly G, Colombel JF, Capron M. Circulating growthregulated oncogene a contributes to neutrophil priming and interleukin-8-directed mucosal recruitment into chronic lesions of patients with Crohn's disease. Eur Cytokine Netw 1998; 9: 647-653.

22. Vercellotti GM, Yin HQ, GustaLson KS, Nelson RD, Jacob HS. Platelet activating factor primes neutrophil responses to agonists: role in promoting neutrophilmediated endothelial damage. Blood 1988; 71: 11001107.

23. Samanta SK, Oppenheim JJ, Matsushima K. Interleukin 8 (monocyte-derived neutrophil chemotactic factor) dynamically regulates its own receptor expression on human neutrophils. J Biol Chem 1990; 265: 183-189.

24. Cummings CJ, Martin TR, Frevert CW, et al. Expression and function of the chemokine receptors CXCR1 and CXCR2 in sepsis. J Immunol 1999; 162: 2341-2346.

25. Nakamura H, Fujishima S, Waki Y, et al. Priming of alveolar macrophages for interleukin-8 production in patients with idiopathic pulmonary fibrosis. $\mathrm{Am}$ J Respir Crit Care Med 1995; 152: 1579-1586.

26. Car BD, Meloni F, Luisetti M, Semenzato G, Gialdroni-Grassi G, Walz A. Elevated IL-8 and MCP-1 in the bronchoalveolar lavage fluid of patients with idiopathic pulmonary fibrosis and pulmonary sarcoidosis. Am Rev Respir Crit Care Med 1994; 149: 655-659.

27. Villard J, Dayer-Pastore F, Hamacher J, Aubert JD, Schlegel-Haueter S, Nicod LP. GRO alpha and interleukin-8 in pneumocystis carinii or bacterial pneumonia and adult respiratory distress syndrome. Am J Respir Crit Care Med 1995; 152: 1549-1554.

28. Kettritz R, Gaido ML, Haller H, Luft FC, Jenette CJ, Falk RJ. Interleukin-8 delays spontaneous and tumor necrosis factor-a-mediated apoptosis of human neutrophils. Kidney International 1998; 53: 84-91.

29. Hammond MEW, Lapointe GR, Feucht PH, et al. IL-8 induces neutrophil chemotaxis predominantly via Type I IL-8 receptors. J Immunol 1995; 155: 1428 1433.

30. Jones SA, Wolf M, Qin SX, Mackay CR, Baggiolini M. Different functions for the IL-8 receptors (IL-8-R) of human neutrophil leukocytes: NADPH oxidase and phospholipase D are activated through IL-8R-1 but not IL-8-R2. Proc Natl Acad Sci USA 1996; 93: 66826686.

31. Jones SA, Dewald B, Clarke-Lewis I, Baggiolini M. Chemokine receptor antagonists that discriminate between Interleukin-8 receptors. J Biol Chem 1997; 272: 16166-16169. 